Proc Indian Natn Sci Acad $\mathbf{8 1}$ No. 1 February 2015 Special Issue, pp. 251-255

(c) Printed in India.

DOI: $10.16943 /$ ptinsa/2015/v81i1/48078

\title{
Cooling of Neutron Star Including Magnetic Interaction
}

\author{
KAUSIK PAL \\ Department of Physics, Serampore College, Serampore 712 201, India
}

(Received on 23 April 2014; Accepted on 12 August 2014)

\begin{abstract}
In this work we calculate the neutrino mean free path (MFP) and neutrino emissivity with non-Fermi liquid (NFL) corrections of degenerate quark matter. Subsequently the role of these NFL corrections on the cooling of neutron star has been demonstrated. The cooling curve shows moderate enhancement due to this NFL corrections.
\end{abstract}

Key Words : Quark Matter; Neutron Star; Non-Fermi Liquid; Magnetic Interaction.

\section{Introduction}

Understanding the properties of the hot and dense ultrarelativistic plasma has been at the forefront of contemporary research for the past few decades. Such studies are important for the understanding of the properties of the astrophysical objects like neutron stars and pulsars. At the core of neutron stars density may go up to $5 \sim 6$ times the normal nuclear matter density. It would be more appropriate to describe the core of such dense stars as degenerate quark matter which is our main interest in the present paper (Boyanovsky and De Vega, 2001a,b; Pal and Dutt-Mazumder, 2011).

In ideal Fermi liquid calculations, like in degenerate electron matter one considers only the chargecharge or electric interaction and the current-current or magnetic interaction is usually neglected while calculating various physical quantities. This is justified in a regime where the velocity of the fermions (electrons or quarks) are small, as in this kinematic regime the magnetic interaction is suppressed in powers of $(v / c)^{2}$. The scenario, however, changes for dense plasma where the constituents like electrons or quarks are moving with a velocity close to the velocity of light. In such cases, the magnetic interaction becomes important. Hence, in dealing with dense plasma, one has to retain both electric and magnetic interactions mediated by the exchange of longitudinal and transverse gauge bosons respectively. In fact, the transverse

*Author for Correspondence : E-mail: kausik.sinp@gmail.com 
interactions, due to its infrared sensitivity, may become more important than its longitudinal counterpart in this kinematic regime. This characteristic behavior has a a non-trivial origin residing in the analytical structure of the Fermion-self energy close to the Fermi surface (Boyanovsky and De Vega, 2001a,b; Pal and Dutt-mazumder, 2011; Schäfer and Schwenzer, 2004; Manuel, 2000; Gerhold et al., 2004; Gerhold and Rebhan, 2005).

In the next section we see the effect of such non-Fermi liquid (NFL) corrections in quantities like neutrino emissivity $(\epsilon)$, neutrino mean free path (MFP) and specific heat $\left(C_{v}\right)$. Equipped with these results, we have studied the cooling behavior of the neutron star.

\section{Mean Free Path, Emissivity and Cooling Behavior}

The neutrino mean free path is determined by the quark-neutrino interaction via weak processes, which are given by (Schäfer and Schwenzer, 2004)

$$
d \rightarrow u+e^{-}+\bar{\nu}_{e} ; \quad u+e^{-} \rightarrow d+\nu_{e}
$$

Now, the neutrino mean free path is related to the total interaction rate due to neutrino emission averaged over the initial quark spins and summed over the final state phase space and spins. It is given by (Pal and Dutt-Mazumder, 2011; Sarkar et al., 2013)

$$
\begin{aligned}
\frac{1}{l_{\text {mean }}\left(E_{\nu}, T\right)} & =\frac{g}{2 E_{\nu}} \int \frac{d^{3} p_{d}}{(2 \pi)^{3}} \frac{1}{2 E_{d}} \int \frac{d^{3} p_{u}}{(2 \pi)^{3}} \frac{1}{2 E_{u}} \int \frac{d^{3} p_{e}}{(2 \pi)^{3}} \frac{1}{2 E_{e}} \\
& \times(2 \pi)^{4} \delta^{4}\left(P_{d}+P_{\nu}-P_{u}-P_{e}\right)|M|^{2} \\
& \times\left\{n\left(p_{d}\right)\left[1-n\left(p_{u}\right)\right]\left[1-n\left(p_{e}\right)\right]-n\left(p_{u}\right) n\left(p_{e}\right)\left[1-n\left(p_{d}\right)\right]\right\} .
\end{aligned}
$$

Here, $|M|^{2}$, the squared invariant amplitude averaged over initial $d$ quark spin and summed over final spins of $u$ quark and electron, is given by (Pal and Dutt-mazumder, 2011; Sarkar et al., 2013)

$$
|M|^{2}=\frac{1}{2} \sum_{\sigma_{u}, \sigma_{d}, \sigma_{e}}\left|M_{f i}\right|^{2}=64 G^{2} \cos ^{2} \theta_{c}\left(P_{d} \cdot P_{\nu}\right)\left(P_{u} \cdot P_{e}\right)
$$

The mean free path at leading order in $T / \mu$ is reduced to (Pal and Dutt-Mazumder, 2011; Sarkar et al., 2013)

$$
\frac{1}{l_{\text {mean }}}=\frac{3 C_{F} \alpha_{s}}{\pi^{4}} G^{2} \cos ^{2} \theta_{c} \mu_{d} \mu_{u} \mu_{e} \frac{\left(E_{\nu}^{2}+\pi^{2} T^{2}\right)}{\left(1+e^{-\beta E_{\nu}}\right)}\left[1+\frac{C_{F} \alpha_{s}}{3 \pi} \ln \left(\frac{m_{D}}{T}\right)\right]^{2} .
$$

To derive the above Eq. (4) we use the modified dispersion relation ( $\mathrm{Pal}$ and Dutt-Mazumder, 2011; Sarkar et al., 2013)

$$
\frac{d p(\omega)}{d \omega} \simeq\left(1-\frac{\partial}{\partial \omega} \operatorname{Re} \Sigma(\omega)\right) \frac{E_{p}(\omega)}{p(\omega)}=\left[1+\frac{C_{F} \alpha_{s}}{3 \pi} \ln \left(\frac{m_{D}}{T}\right)\right] \frac{E_{p}(\omega)}{p(\omega)}
$$


where we denote the single particle energy $E_{u(d)}$ as $\omega$. The one-loop quark self-energy $\Sigma$ is (Manuel, 2000; Gerhold et al., 2004)

$$
\Sigma=\frac{g^{2} C_{F}}{12 \pi^{2}}(\omega-\mu) \ln \left(\frac{m_{D}}{\omega-\mu}\right)+i \frac{g^{2} C_{F}}{12 \pi}|\omega-\mu| .
$$

Here $m_{D}$ represents the Debye mass.

Once the mean free path is known, we can easily calculate the neutrino emissivity $(\epsilon)$ by evaluating the integral

$$
\begin{aligned}
\epsilon & =\int \frac{d^{3} p_{\nu}}{(2 \pi)^{3}} \frac{E_{\nu}}{l_{\text {mean }}\left(-E_{\nu}, T\right)} \\
& \approx \frac{457}{630} G^{2} \cos ^{2} \theta_{c} \alpha_{s} \mu_{u} \mu_{d} \mu_{e} T^{6}\left[1+\frac{C_{F} \alpha_{s}}{3 \pi} \log \left(\frac{m_{D}}{T}\right)\right]^{2} .
\end{aligned}
$$

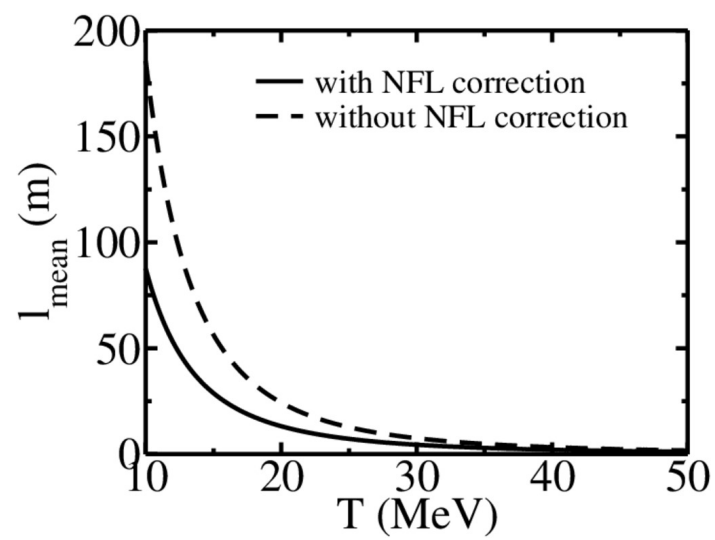

A

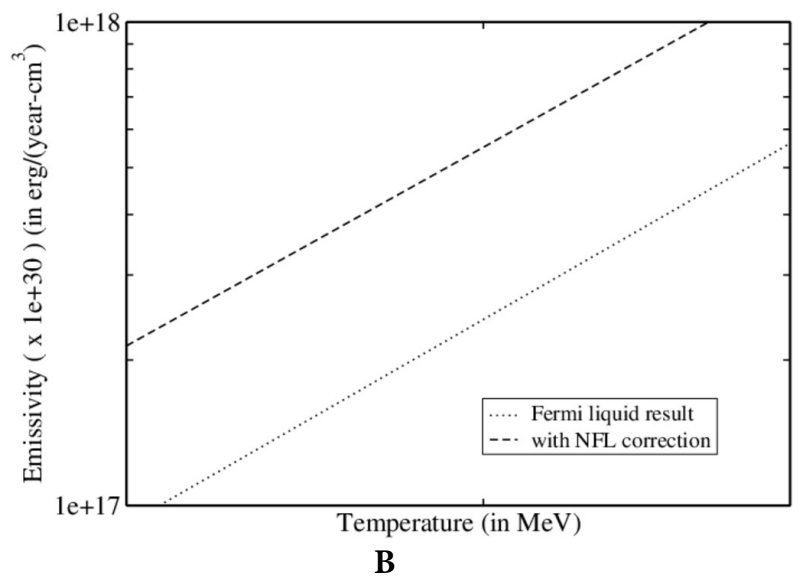

B

Fig. 1: Neutrino mean free path (A) and neutrino emissivity (B) of degenerate quark matter

In Fig. 1, we find that the anomalous logarithmic term reduces the value of the mean free path appreciably at low temperature while at higher temperature it tends to merge (Pal and Dutt-Mazumder, 2011). For the emissivity, the anomalous logarithmic terms dominate in the relevant temperature range and exceed the Fermi liquid result considerably (Schäfer and Schwenzer, 2014; Sarkar et al., 2013). This reduced mean free path or enhanced emissivity is expected to influence the cooling of neutron star.

To analyze the cooling of the star the specific heat capacity of the quark matter core needs to be taken into consideration along with the emissivity via the cooling equation (Boyanovsky and De Vega, 2001a,b; Schäfer and Schwenzer, 2004)

$$
C_{v}(T) \frac{d T}{d t}=-\epsilon(T)
$$


where $C_{v}$ is the specific heat at constant volume and baryon number, $\epsilon$ is the emissivity, $t$ is time, $T$ is the temperature.

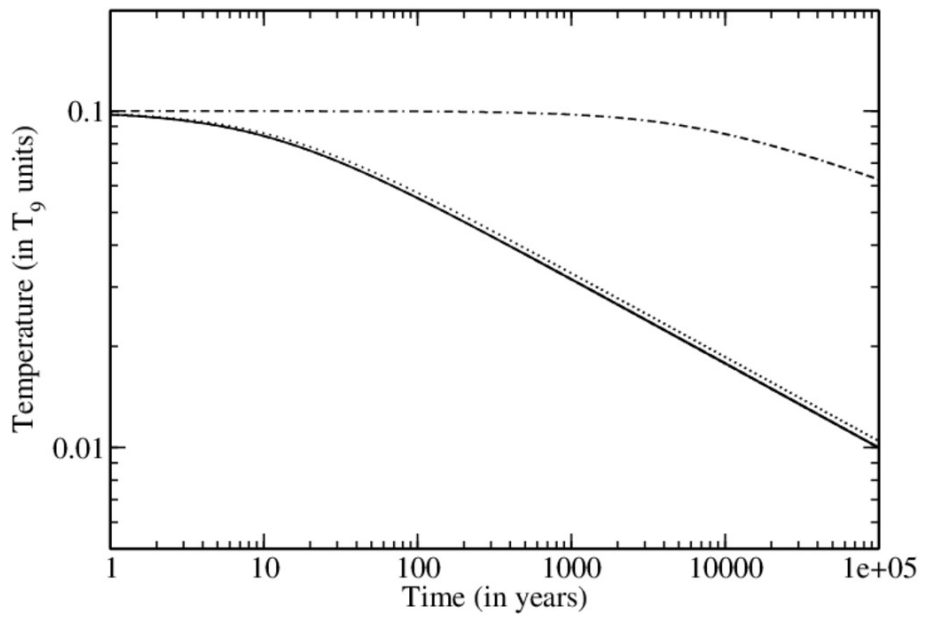

Fig. 2: The cooling behavior of neutron star with core as neutron matter and degenerate quark matter. The dotted line represents the Fermi liquid result, the solid line gives the result including non-Fermi liquid correction. The dash-dotted line represents for purely neutron matter

In Fig. 1A, we note that there is a considerable decrease in MFP of neutrinos due to NFL corrections over the Fermi liquid result. This difference in the MFP between NFL and Fermi liquid corrections also leads to modest increase in the emissivity of the neutrinos for the two cases as displayed in Fig. 1B. This in turn causes the cooling of neutron star to become significantly faster in case of NFL as compared to the Fermi liquid result as shown in Fig. 2 (Schäfer and Schwenzer, 2004; Sarkar et al., 2013).

\section{Summary and Conclusion}

In this work, we have calculated the MFP and neutrino emissivity including NFL corrections of degenerate quark matter. It is seen, both the MFP and the emissivity receives logarithmic corrections. Equipped with these results we have examined the cooling behavior of a neutron star.

\section{Acknowledgments}

I am indebted to Late Prof. A.K. Dutt-Mazumder for suggesting this topic and for many stimulating and enthusiastic discussions motivated me to carry forward my research work.

\section{References}

1. Boyanovsky D and De Vega HJ (2001a) The specific heat of normal, degenerate quark matter: Non-Fermi liquid corrections, Phys Rev D63 114028 
2. Boyanovsky D and De Vega HJ (2001b) Non-Fermi liquid aspects of cold and dense QED and QCD: Equilibrium and Non-Equilibrium, Phys Rev D63 034016

3. Pal K and Dutt-Mazumder AK (2011) Non-Fermi liquid corrections to the neutrino mean free path in dense quark matter, Phys Rev D84 034004

4. Schäfer T, Schwenzer K (2004) Neutrino emission from ungapped quark matter, Phys Rev D70 114037

5. Manuel C (2000) Dispersion relations in ultradegenerate relativistic plasmas, Phys Rev D62 076009

6. Gerhold A, Ipp A and Rebhan A (2004) Non-Fermi liquid specific heat of normal degenerate quark matter, Phys Rev D70 105015

7. Gerhold A and Rebhan A (2005) Fermionic dispersion relations in ultradegenerate relativistic plasmas beyond leading logarithmic order, Phys Rev D71 085010

8. Sarkar S, Pal K and Dutt-Mazumder AK (2013) Role of magnetic interaction in dense plasma, Avdances in High Energy Physics 2013530895. 